\title{
Polymorphous Low-grade Adenocarcinoma of Base of the Tongue: A Case Report and Review of Literature of an Orphan Disease
}

\author{
Anil Khurana ${ }^{1}$, Vijay Kumar ${ }^{2}$, Paramjeet Kaur ${ }^{3}$, Sumiti Gupta ${ }^{4}$, Ashok Chauhan ${ }^{5}$
}

\begin{abstract}
Here, we are presenting a case of polymorphous low-grade adenocarcinoma (PLGA) of base of tongue, which is a malignancy with low-grade aggressiveness, less metastatic potential in a 76-year-old male with swelling on the left side of the neck for 2 weeks. The patient received a radical external beam radiation therapy on face and neck. Now, the patient is disease free and is on follow-up a year after the treatment.

Keywords: Chemotherapy, Low-grade tumors, Minor salivary glands, Radiation therapy, Surgery.

International Journal of Head and Neck Surgery (2020): 10.5005/jp-journals-10001-1385
\end{abstract}

\section{INTRODUCTION}

Polymorphous low-grade adenocarcinoma (PLGA) is a tumor of minor salivary glands with indolent course with fewer incidences in oral cavity and oropharynx. Surgery is the primary treatment modality even though the role of radiotherapy and chemotherapy is ill-defined.

\section{Case Description}

A 76-year-old male from a rural area presented with complaints of swelling on the left side of neck for two weeks. The patient was apparently normal fortnight back, and then, he noticed a tiny swelling over the left side of the neck, which was gradually progressive in nature to attend the present size of marble. Swelling was not associated with any pain. The patient also gave a history of difficulty in swallowing since one week, which was gradually progressive in nature and it was more associated with solid foods in comparison with semisolid and liquid foods. The patient did not have any history of decreased appetite and weight loss, and neither hoarseness nor hemoptysis. The patient is a chronic smoker since 40 years, and he used to smoke 10-12 bidis per day, but he was a nonalcoholic. There is no significant past history, family history, and medical history. On general physical examination, patient was well built and nourished with well orientation to time, place, and person. On inspection, oral cavity and oropharynx were normal. On palpation, oral cavity was normal whereas there was induration felt over the base of the tongue whose lower extent cannot be defined. On indirect laryngoscopy, ulceroprolifertive growth was seen involving the base of the tongue with extension into the vallecula and epiglottis. On neck examination, there was a level II solitary lymph node on the right side measuring about $2 \mathrm{~cm} \times 1 \mathrm{~cm}$, which was firm in consistency, nontender, and mobile in both horizontal and vertical directions. A systemic examination of cardiovascular, respiratory, central nervous system, and abdomen was normal. Laboratory blood investigations such as complete hemogram profile, renal function tests, and liver function tests were normal. Chest $\mathrm{X}$-ray was normal (Fig. 1). Soft tissue neck lateral view demonstrates growth over the base of the tongue (Fig. 2). Contrast-enhanced computed tomography (CECT) of oropharynx

\begin{abstract}
1,5 Department of Radiotherapy, Pt BD Sharma Post Graduate Institute of Medical Sciences, Rohtak, Haryana, India

${ }^{2}$ Department of Radiotherapy, HCG Cancer Centre, Bengaluru, Karataka, India

${ }^{3}$ Department of Radiation Oncology, Pt BD Sharma Post Graduate Institute of Medical Sciences, Rohtak, Haryana, India

${ }^{4}$ Department of Pathology, Pt BD Sharma Post Graduate Institute of Medical Sciences, Rohtak, Haryana, India
\end{abstract}

Corresponding Author: Paramjeet Kaur, Department of Radiation Oncology, Pt BD Sharma Post Graduate Institute of Medical Sciences, Rohtak,Haryana,India,Phone:+918901387991,e-mail:drparamjitkaur@ rediffmail.com

How to cite this article: Khurana A, KumarV, Kaur P, et al. Polymorphous Low-grade Adenocarcinoma of Base of the Tongue: A Case Report and Review of Literature of an Orphan Disease. Int J Head Neck Surg 2020;11(4):71-74.

Source of support: Nil

Conflict of interest: None

and neck demonstrates a moderately enhancing soft tissue density mass lesion with ill-defined margins, which is seen involving right posterior base of the tongue extending to floor of the mouth where it is forming an ill-defined enhancing mass lesion. An approximate size of the lesion is $3 \times 2.8 \times 2.6 \mathrm{~cm}$ ( AP $\times$ Transverse $\times C C)$. This lesion is just crossing the midline. Inferiorly, the lesion is extending up to the hyoid bone and touching its superior surface with no obvious evidence of erosion; mass is extending into preepiglottic space and involving the lingual surface of epiglottis, and it is also extending into adjacent right lateral oropharyngeal wall of adjacent oropharynx (Fig. 3). On CECT scan, an enlarged necrotic lymph node with the size of $2 \times 1.8 \mathrm{~cm}$ is seen in the right upper jugulodigastric level (Fig. 4), and centrilobular emphysematous changes are seen in the visualized bilateral lung fields along with minimal fibrosis noted in bilateral apex. A pulmonologist opinion was taken regarding the findings in the CECT scan; as the patient was asymptomatic, there was also no history of antitubercular drug intake and no members in the family suffering from pulmonary Koch's, so the patient was kept on a regular follow-up. Histopathological examination 


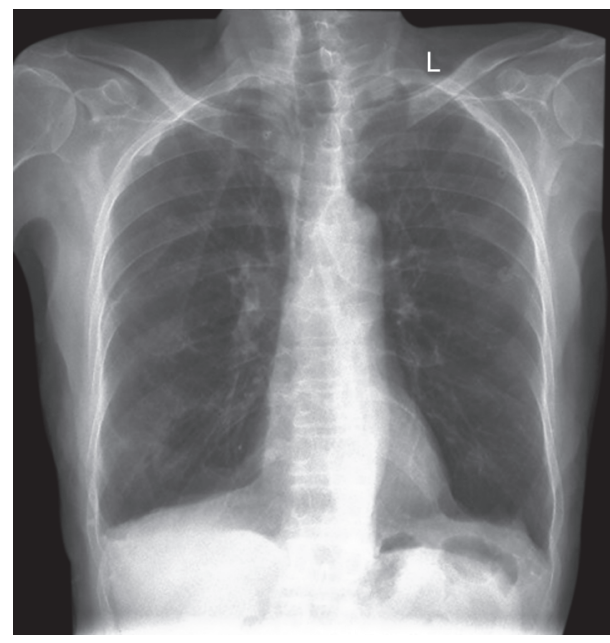

Fig. 1: Normal chest X-ray

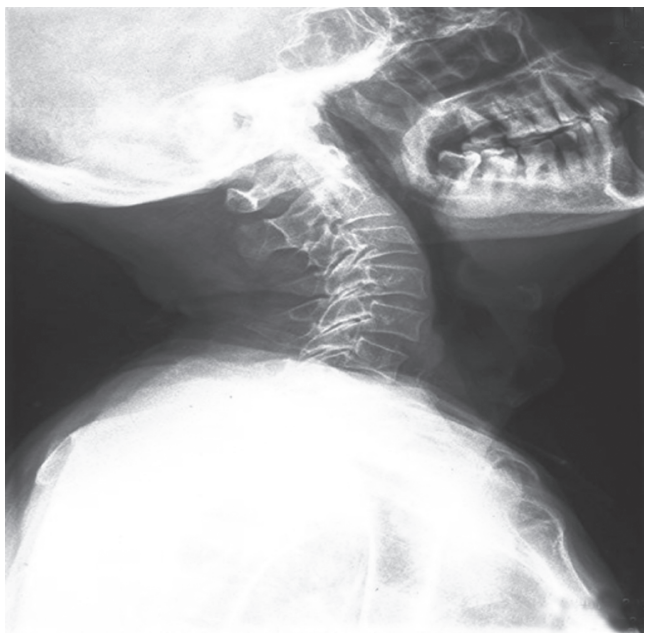

Fig. 2: Soft tissue neck lateral view demonstrates growth over the base of the tongue
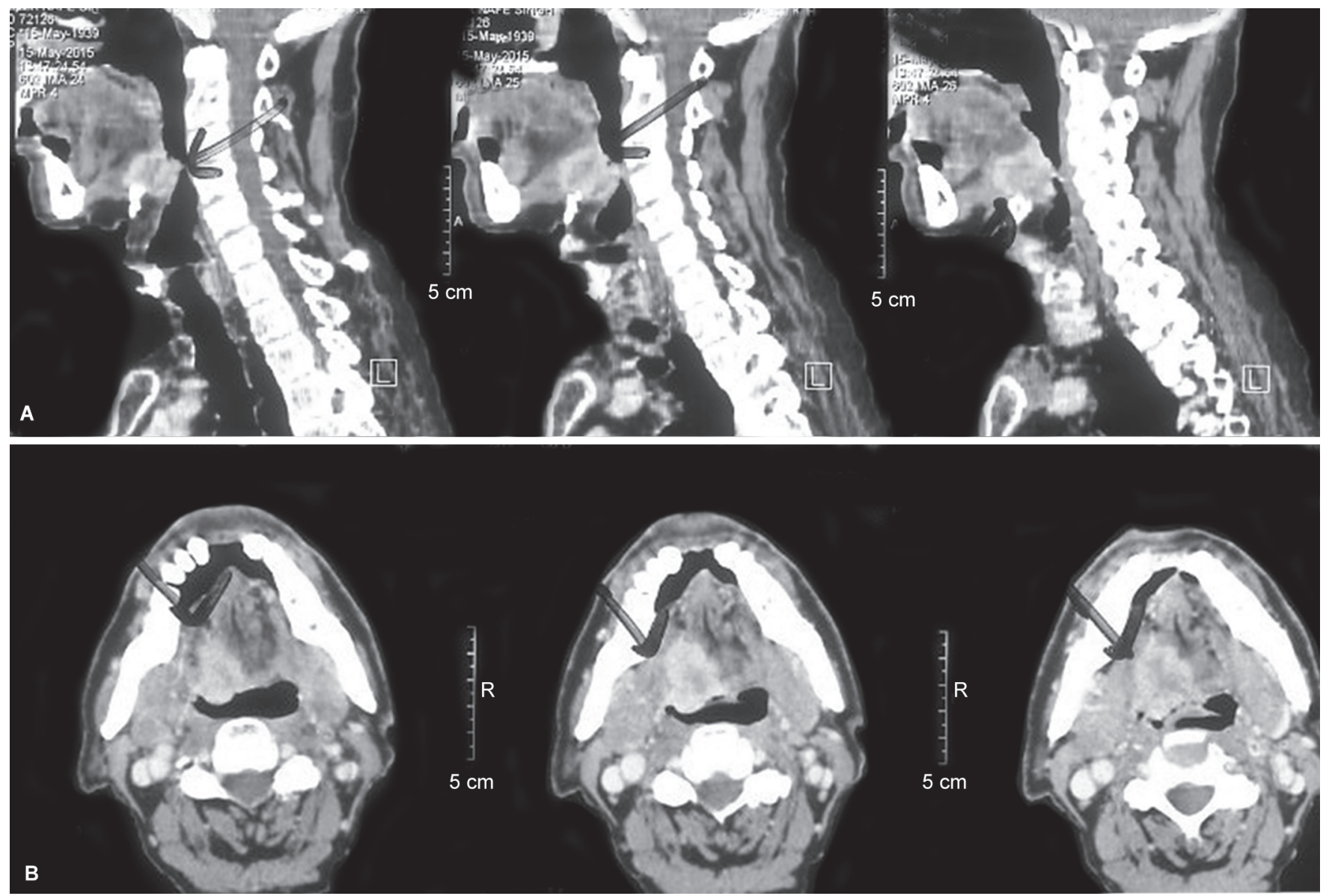

Figs $3 \mathrm{~A}$ and B: Contrast-enhanced computed tomography of oropharynx and neck demonstrates a moderately enhancing soft tissue density mass lesion with ill-defined margins, which is seen involving right posterior base of the tongue extending to floor of the mouth where it is forming an ill-defined enhancing mass lesion

of incision biopsy from the base of the tongue demonstrated PLGA with origin from the minor salivary gland. There was not any perineural invasion, and on immunohistochemical profile, epithelial membrane antigen (EMA) was positive (diffusely strong), p63 (irregular positivity), whereas smooth muscle actin (SMA) and S-100 were negative (Fig. 5). On the basis of histopathological and immunohistochemical profile, the diagnosis of PLGA of base of the tongue with origin from the minor salivary gland was made. The patient planned to undergo a radical external beam radiation therapy with dose schedule of 66 Gy in 33 fractions with dose rate of 200 cGy per day (conventional) by bilateral parallel opposed fields on face and neck with spinal cord sparing after 44 Gy to respect 

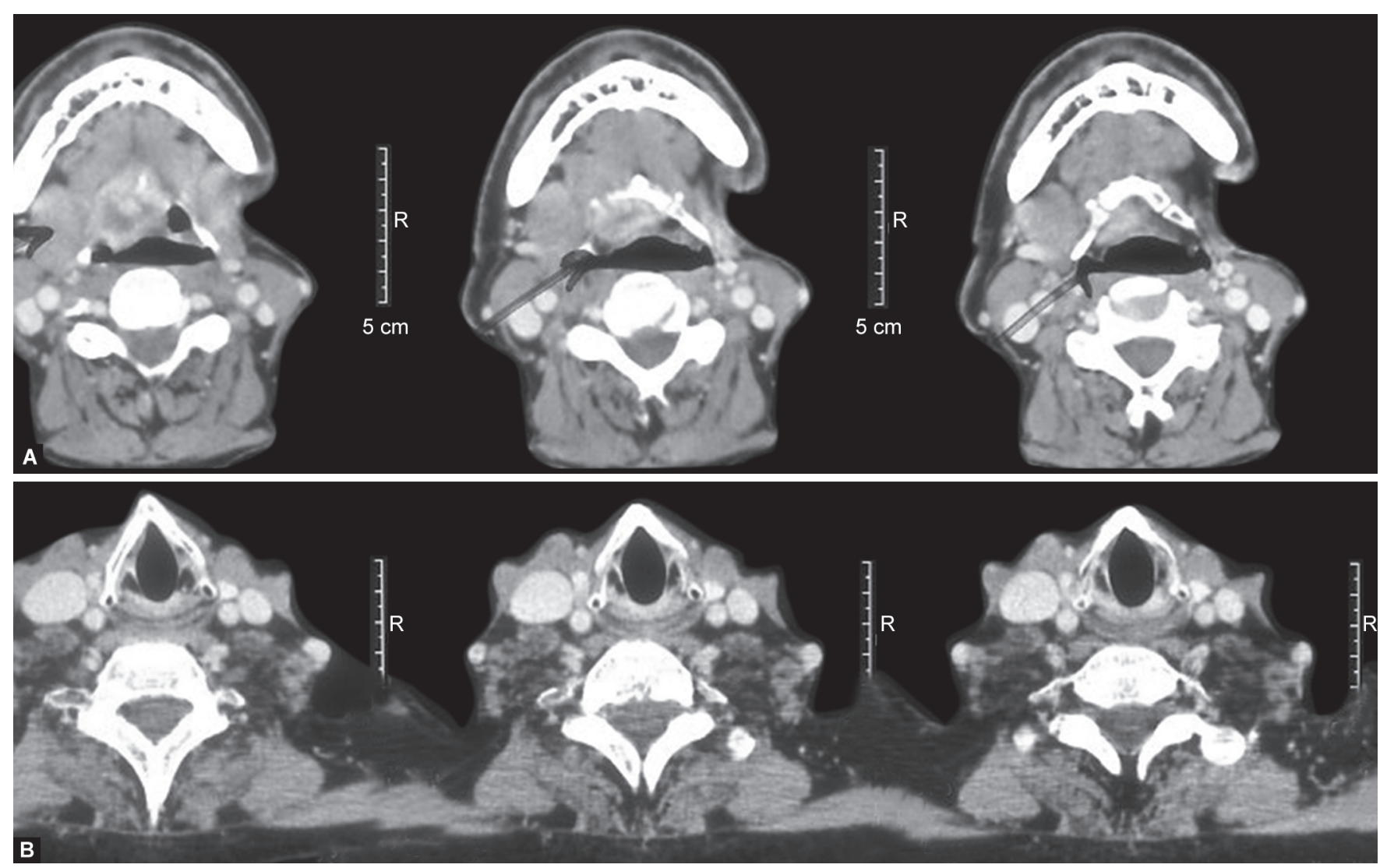

Figs 4A and B: Contrast-enhanced computed tomography scan demonstrating enlarged necrotic lymph node with the size of $2 \times 1.8 \mathrm{~cm}$, which is seen in the right upper jugulodigastric level

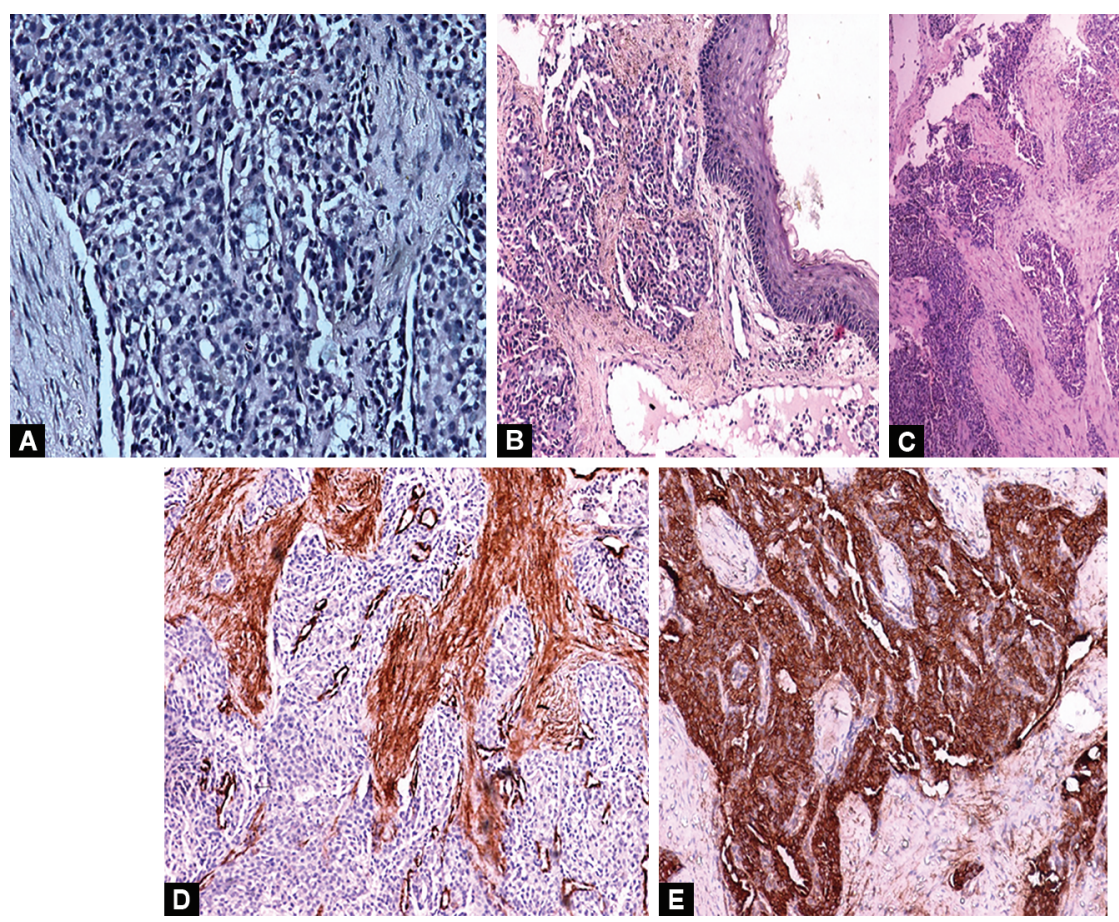

Figs 5 A to E: Photomicrographs showing: (A to C) Low-grade adenocarcinoma (20, 10, and $4 \times$ ); (D) Tumor cells with smooth muscle actin positivity (10X); (E) Tumor cells with epithelial membrane antigen positivity (10X) 
the normal tissue tolerance dose of spinal cord ( $\mathrm{TD}_{5 / 5}$ volume) by cobalt-60 teletherapy machine. Now, the patient is disease free clinically and radiologically and on follow-up a year after treatment.

\section{Discussion}

History of PLGA dates back to 1983 when Freedman and Lumerman described it as a distinct entity, and later on, Evans and Batsakis coined the term PLGA. ${ }^{1}$ Polymorphous low-grade adenocarcinoma is a tumor of minor salivary glands. ${ }^{2}$ Histopathologically, adenocarcinoma of tongue constitutes less than $2 \%$ of all malignancies. ${ }^{3}$ But there are some exceptions such as tumors with predominant papilliferous arrangement, which have an aggressive clinical behavior, so it is usually classified as papillary cystadenocarcinomas. ${ }^{4}$

Mucoepidermoid carcinoma is the most common tumor of the minor salivary glands followed by the PLGA, which is the second most common tumor. ${ }^{5}$

Various nonminor salivary gland sites include palate, upper lip, buccal mucosa, posterior tongue, retromolar trigone, paranasal sinuses, major salivary glands, the breast, paranasal sinuses, the skin and orbit, and vulva and vagina. ${ }^{6}$

It is usually seen in the fifth to seventh decades of life, but there are some exceptions in which adolescent age group has also been reported in the literature. It is more common in the females when compared to male with a ratio of $2-4 \cdot 6: 1 .{ }^{6}$ But Gupta et al. commented that the disease is more common in females with a male to female ratio of 1:1.02-1:20.

Castle et al. did a retrospective clinicopathologic study of 164 cases from 1970 to 1994 , in which there were more number of female patients in comparison with males (109 women and 55 men). ${ }^{8}$ Polymorphous low-grade adenocarcinoma originates from the intercalated ducts and reserved cells. Polymorphous low-grade adenocarcinoma usually presents as an asymptomatic slow-growing painless mass, which is occasionally associated with bleeding or discomfort. ${ }^{9}$

Differential diagnosis of PLGA consists of pleomorphic adenoma and cystic adenocarcinoma. Gross feature of pleomorphic adenoma consists of well circumscribed margins with capsule formation, whereas microscopically, it is characterized by chondromyxoid matrix and plasmacytoid cells. Adenoid cystic carcinoma is characterized microscopically by the presence of cribriform and tubular structures with double-layered ducts composed of angular hyperchromatic cells. PLGA is characterized by lack of unique immunohistochemical markers. ${ }^{10}$

Epithelial tumor markers, S-100 protein, and vimentin are expressed by tumor cells. ${ }^{11}$

Regional and distant metastasis rates in PLGA are rare; regional metastasis ranges between $5 \%$ and $15 \%$, whereas distant metastasis ranges from $0.6 \%$ to $7.5 \% .{ }^{10} \mathrm{Kim}$ et al. reported a role of transoral robotic surgery in the treatment of PLGA of the base of the tongue without any complication of aspiration or dysphagia. ${ }^{10}$ Gupta et al. reported a case of PLGA of palate with extension into the maxillary sinus and nasal cavity, which was treated by subtotal maxillectomy with good success rate. ${ }^{7}$

Neck dissection is usually indicated in the patients who are present with radiological or clinical evidence of lymph nodes, ${ }^{12,13}$ whereas elective neck dissection is indicated in PLGA of the base of the tongue.

The benefit of radiation therapy in the management of PLGA is not well defined. It is indicated postoperatively in case of positive or even in close surgical margins. ${ }^{7}$ It is also indicated in adjuvant setting in the patients with cervical metastasis. ${ }^{7}$ Makita et al. assessed the role of proton beam therapy on a patient with a dose of 70.4 Gy in 32 fractions and intra-arterial chemotherapy consisting of the weekly cisplatin. Stable disease was maintained for 2 years without evidence of new metastasis. There was no hematological toxicity or neurological toxicity, but there was grade III dermatitis and to mucositis. ${ }^{14}$

\section{CONCLUSION}

Polymorphous low-grade adenocarcinoma of base of the tongue is a tumor with low-grade aggressiveness and less incidence of distant metastasis. The main diagnostic method for the diagnosis is biopsy with a lack of characteristic immunohistochemical markers. The main treatment modality of this rare disease is surgery with ill-defined role of the radiation therapy and chemotherapy, even though there are experiences with rare treatment modalities such as proton beam therapy.

\section{References}

1. Majumdar S, Roy JG, Uppala D. Polymorphous Low Grade Adenocarcinoma: A Report of Two Cases. Oral Maxillofac Pathol J 2014;5(2):520-522.

2. Hannen EJ, Bulten J, Festen J, et al. Polymorphous low grade adenocarcinoma with distant metastases and deletions on chromosome 6q23-qter and 11q23-qter: a case report. J Clin Pathol 2000 Dec;53(12):942-945. DOI: 10.1136/jcp.53.12.942.

3. Attner $P, D u J$, Näsman $A$, et al. Human papillomavirus and survival in patients with base of tongue cancer. Int J Cancer 2011;128:2892-2897. DOI: 10.1002/ijc.25625.

4. Brocheriou C. Polymorphous low-grade adenocarcinoma of the minor salivary glands: seven cases. Arch Anat Cytol Pathol 1992;40: 6672.

5. Waldron CA, el-Mofty SK, Gnepp DR. Tumors of the intraoral minor salivary glands: a demographic and histologic study of 426 cases. Oral Surg Oral Med Oral Pathol 1988 Sep;66(3):323-333. DOI: 10.1016/0030-4220(88)90240-X.

6. Olusanya AA, Akadiri OA, Akinmoladun VI, et al. Polymorphous low grade adenocarcinoma: literature review and report of lower lip lesion with suspected lung metastasis. J Maxillofac Oral Surg 2011 Mar;10(1):60-63. DOI: 10.1007/s12663-011-0185-1.

7. Gupta S, Kumar CA, Raghav N, et al. Polymorphous Low-grade Adenocarcinoma of the Palate: Report of a Case and Review of Literature. Int J Head Neck Surg January-April 2011;2(1):57-60. DOI: 10.5005/jp-journals-10001-1049.

8. Castle JT, Thompson LD, Frommelt RA, et al. Polymorphous low grade adenocarcinoma: a clinicopathologic study of 164 cases. Cancer 1999 Jul 15;86(2):207-219. DOI: 10.1002/(SICI)10970142(19990715)86:2<207::AID-CNCR4>3.0.CO;2-Q.

9. Kulkarni M, Gabhane M, Mahajan A, et al. Polymorphous Low Grade Adenocarcinoma: A Case Report. IJDS June 2011;2(3):23-25.

10. Kim JH, Hyun CL, Lim GC. Polymorphous low-grade adenocarcinoma of the tongue base treated by transoral robotic surgery. Case Rep Otolaryngol 2015;2015:981436. DOI: 10.1155/2015/981436.

11. Anderson C, Krutchkoff D, Peterson C, et al. Polymorphous low grade adenocarcinoma of minor salivary glands: a clinicopathologico and comparative immunohistochemical study. Modern pathol 1990;3:76-82.

12. Abu El-Naaj I, Leiser $Y$, Wolff A, et al. Polymorphous low grade adenocarcinoma: case series and review of surgical management. J Oral Maxillofac Surg 2011;69(7):1967-1972. DOI: 10.1016/ j.joms.2010.10.010.

13. Pogodzinski MS, Sabri AN, Lewis JE, et al. Retrospective study and review of polymorphous low-grade adenocarcinoma. Laryngoscope 2006;116(12):2145-2149. DOI: 10.1097/01.mlg.0000243200.35033.2b.

14. Makita C, Nakamura T, Takayama K, et al. Proton beam therapy and continuous intra-arterial chemotherapy for polymorphous lowgrade adenocarcinoma in the hard palate. Case Rep Oncol 2013 Jan;6(1):66-71. DOI: 10.1159/000346840. 